\title{
Holographie spectro-temporelle
}

\author{
J.-L. Le Gouët \\ Laboratoire Aimé Cotton, UPR 3321 du CNRS, Centre Scientifique d'Orsay, \\ bâtiment 505, 91405 Orsay cedex, France
}

\begin{abstract}
Résumé : L'utilisation d'un matériau photosensible sélectif en longueur d'onde permet d'étendre à la dimension spectro-temporelle les concepts de l'holographie, d'habitude restreints aux seules dimensions spatiales. Après avoir examiné le principe de l'enregistrement dans un tel matériau nous dressons un bref inventaire de quelques expériences réalisées ou projetées en vue d'applications au stockage et au traitement optique de l'information.
\end{abstract}

\section{INTRODUCTION}

Alors que la photographie enregistre la distribution d'intensité de la lumière, c'est une cartographie complète ( holo $»=$ tout entier) du champ électromagnétique que réalise l'holographie par le biais d'une gravure interférentielle. Encore enregistre-t-on seulement une répartition spatiale. Celle-ci suffit à décrire complètement un champ monochromatique mais le matériau photosensible, en général peu sélectif en longueur d'onde, est impuissant à enregistrer l'information contenue dans la distribution spectro-temporelle d'un champ polychromatique.

Le présent exposé traite d'holographie réalisée dans des matériaux tout différents, capables de sélectivité en longueur d'onde à l'échelle microscopique. Avec la dimension spectrale, c'est aussi la dimension temporelle, conjuguée de la première, qui s'ajoute à la cartographie du champ. Ces propriétés se paient par l'inconvénient d'un fonctionnement à très basse température. Quelles applications pourraient s'accommoder d'une telle contrainte? Puisqu'une dimension est ajoutée au volume de stockage, on envisage d'emblée la réalisation de mémoires optiques rapides de grande capacité. D'autre part, les propriétés de traitement optique du signal déjà rencontrées en holographie spatiale méritent d'être étendues au domaine spectro-temporel. Enfin, on cherche à exploiter les fonctionnalités que les hologrammes spectro-temporels partagent avec les modulateurs acousto-optiques, combinant comme eux sélectivité spectrale et spatiale, mais avec des bandes passantes et des produits temps $\times$ bande passante bien supérieurs.

Les notions d'holographie évoquées dans l'introduction sont précisées dans le paragraphe 2. Le paragraphe 3 décrit les mécanismes physiques mis en jeu dans un 
enregistrement sélectif en longueur d'onde. Dans le paragraphe 4 sont discutées des applications où des hologrammes monochromatiques sont enregistrés à différentes longueurs d'onde. La dimension temporelle est introduite dans le paragraphe 5 et les potentialités en termes de traitement optique spectro-temporel sont présentées dans le paragraphe 6.

Nous laisserons de côté la propriété de sélectivité de Bragg qui, dans le cas de l'holographie en volume, permet de réaliser une certaine discrimination spectrale [1]. Celle-ci repose sur un filtrage en longueur d'onde interférentiel lié à l'épaisseur du réseau enregistré dans le matériau photosensible.

\section{ENREGISTREMENT DU CHAMP}

Lors de l'enregistrement d'un hologramme conventionnel, un point $\mathbf{r}$ d'un matériau photosensible est éclairé par les champs monochromatiques objet et référence $a(r)$ et $b(\mathbf{r})$. La gravure effectuée en ce point est proportionnelle au produit $a(\mathbf{r}) b^{*}(\mathbf{r})$. Le signal émis par ce point, lorsqu'il est interrogé par un champ de lecture $\mathbf{c}(\mathbf{r})$, est proportionnel à $a(\mathbf{r}) b^{*}(\mathbf{r}) \mathrm{c}(\mathbf{r})$ (figure 1.a). Cette expression reflète le caractère local de l'émission, dans l'espace des positions : le signal émis au point $r$ est fonction de l'enregistrement effectué en ce point.

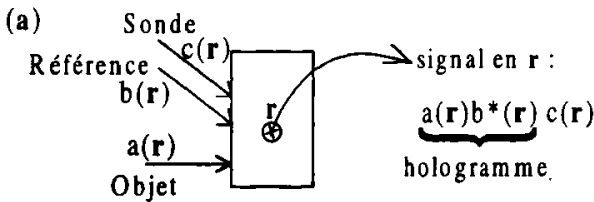

(b)

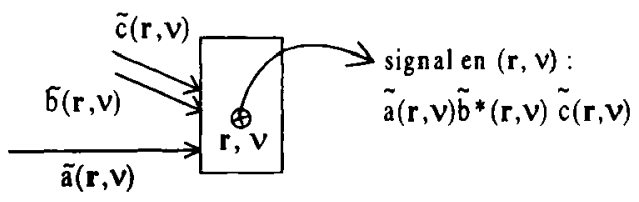

(c)

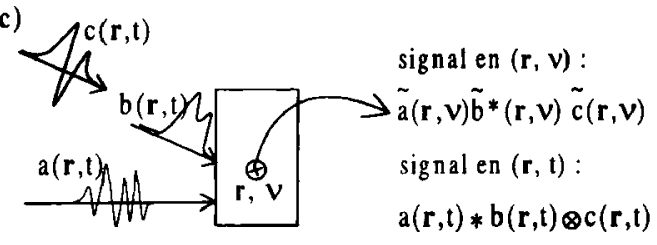

Figure 1 : Holographie conventionnelle, source monochromatique (a). Holographie spectro-spatiale dans un matériau sélectif en fréquence, source polychromatique (b). Holographie spectro-spatiale produite par des champs dépendant du temps (c).

En général la localité ne se manifeste pas dans l'espace des fréquences temporelles. Par exemple, à condition de satisfaire la condition d'accord de phase, on peut fort bien relire à l'aide d'un laser hélium-néon un hologramme gravé par le faisceau vert d'un laser à argon ionisé. Il existe pourtant des matériaux photosensibles où la propriété de localité se rencontre également dans l'espace des fréquences temporelles. Dans un tel matériau sélectif en 
fréquence, il faut, pour définir un point, compléter ses coordonnées spatiales $\mathbf{r}$ par sa fréquence temporelle $v$ (figure 1.b). On peut alors mettre en oeuvre des champs polychromatiques qui au point $(\mathbf{r}, v)$ sont caractérisés par les amplitudes $\tilde{a}(\mathbf{r}, v), \widetilde{b}(\mathbf{r}, v)$, $\widetilde{c}(\mathbf{r}, v)$. La gravure en $(\mathbf{r}, v)$ est proportionnelle a $\tilde{\mathbf{a}}(\mathbf{r}, v) \tilde{b} *(\mathbf{r}, v)$ et la contribution de ce point spatio-spectral au signal holographique est donnée par [2] :

$$
\tilde{s}(\mathbf{r}, v)=\tilde{a}(\mathbf{r}, v) \tilde{b} *(\mathbf{r}, v) \tilde{c}(\mathbf{r}, v)
$$

La composante spectrale du faisceau sonde à la fréquence $v$ est diffractée par la composante de même fréquence du hologramme, et par elle seule.

Les amplitudes spectrales et temporelles $\tilde{\mathrm{f}}(\mathbf{r}, v)$ et $f(r, t)$ des champs lumineux sont reliées entre elles par transformation de Fourier suivant l'équation :

$$
f(\mathbf{r}, t)=\int d v \tilde{f}(\mathbf{r}, v) \exp 2 i \pi v t
$$

Substituant cette relation dans l'équation 1, on exprime l'amplitude temporelle du signal sous la forme :

$$
s(\mathbf{r}, t)=a(r, t) * b(r, t) \otimes c(r, t)
$$

où $*$ et $\otimes$ désignent respectivement la corrélation et la convolution des profils temporels des ondes excitatrices. Si la référence d'écriture $b(r, t)$ et l'onde de lecture $c(r, t)$ sont brèves et se propagent dans la même direction, le signal se réduit à $a(r, t)$, il reproduit fidèlement le profil temporel du champ objet enregistré. En particulier on retrouve, entre l'onde de lecture et le signal, le même décalage qu'entre la référence $b(r, t)$ et l'objet $a(r, t)$. On reconnaît le phénomène d'écho de photon stimulé [3], mis en évidence en 1978 dans le contexte de l'excitation résonnante d'une transition atomique et analogue au processus d'écho de spin rencontré en résonance magnétique nucléaire.

\section{3. « HOLE BURNING » SPECTRAL}

Les hologrammes conventionnels sont en général gravés sous forme de réseaux d'indice dans des matériaux quasi transparents, ce qui permet en principe d'atteindre une efficacité de diffraction élevée. L'holographie spectro-temporelle requiert une sélectivité spectrale que ne présente pas l'indice de réfraction mais que possède en revanche le coefficient d'absorption des matériaux refroidis à très basse température.

Lorsque des chromophores (ions ou molécules) sont dispersés dans un solide, leurs niveaux d'énergie interne sont déplacés par interaction avec l'environnement. Dans un cristal parfait, des centres absorbants, tous disposés identiquement par rapport au réseau cristallin, subiraient tous le même déplacement de niveaux. Leurs raies d'absorption seraient toutes identiques, centrées à la même fréquence, et posséderaient toutes la même largeur appelée largeur homogène et notée $\Gamma_{\text {hom }}$. Dans une matrice réelle, dont le réseau présente du désordre et des défauts, chaque site d'insertion donne naissance à un déplacement spécifique de la fréquence de transition. Les raies d'absorption individuelles, toujours caractérisées par une largeur commune $\Gamma_{\text {hom, }}$ se trouvent distribuées sur un intervalle spectral de largeur $\Gamma_{\text {inh, }}$, 

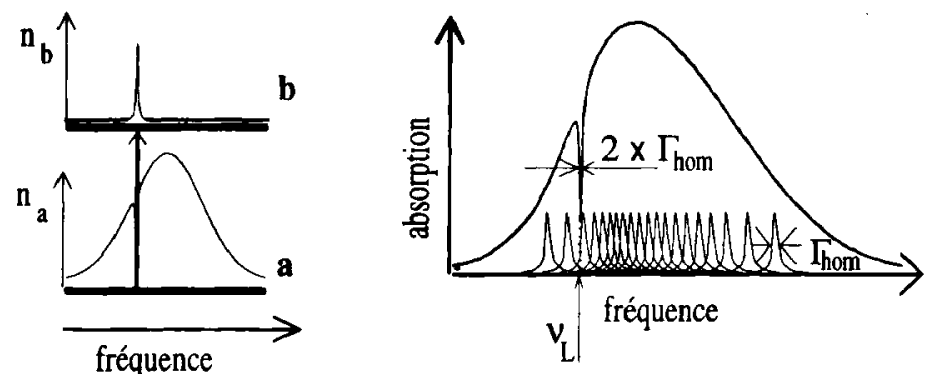

Figure 2 : Des centres absorbants à deux niveaux d'énergie a et b sont excités à la fréquence $v_{\mathrm{L}}$. À gauche: distribution spectrale des populations des deux niveaux après excitation. À droite: modification du profil de la bande d'absorption. On a représenté les raies homogènes de largeur $\Gamma_{\text {hom }}$ dont l'enveloppe constitue la bande de largeur inhomogène $\Gamma_{\text {inh }}$.

appelée largeur inhomogène. À basse température, le spectre inhomogène reste large cependant que les spectres individuels des chromophores dopants s'affinent considérablement. À $10 \mathrm{~K}$, la largeur homogène $\Gamma_{\text {hom }}$ peut être de $10^{3}$ à $10^{9}$ fois plus petite que la largeur inhomogène $\Gamma_{\text {inh }}$.

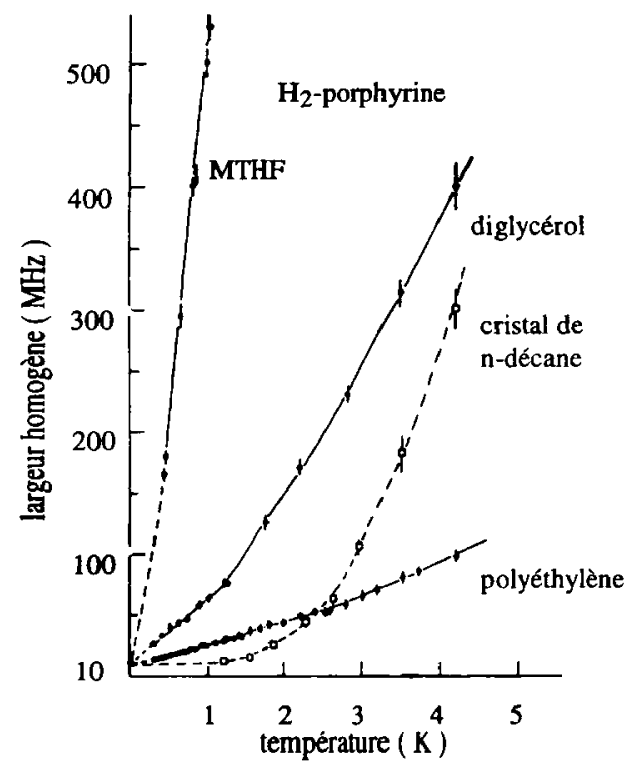

Figure 3 : Élargissement homogène de la raie à $S_{1}-S_{0} 0-0$ de la porphyrine-base dispersée dans différentes matrices organiques

Il a fallu attendre l'invention du laser pour confirmer expérimentalement cette description. En effet la sous-structure homogène d'un spectre d'absorption n'est pas directement accessible. Le profil enregistré par spectroscopie linéaire conventionnelle est toujours affecté par l'élargissement inhomogène. Avec l'avènement du laser ont été inaugurées les techniques de spectroscopie de saturation qui, pour la première fois, ont donné 
accès à $\Gamma_{\text {hom }}$. Il s'agit de processus en deux étapes. Accordé a la fréquence $v_{L}$, un laser monochromatique illumine tout d'abord l'échantillon et porte dans le niveau supérieur de la transition les centres absorbants dont la fréquence coïncident avec $v_{L}$ à $\Gamma_{\text {hom }}$ près. On peut alors analyser, par exemple à travers un Fabry-Perot, le spectre d'émission des atomes ainsi excités. C'est la technique d'affinement de fluorescence ou Fluorescence Line Narrowing. Par ailleurs, le dépeuplement du niveau fondamental et le peuplement corrélatif du niveau excité se traduisent par une réduction de l'absorption sur l'intervalle spectral atteint par le laser. C'est le phénomène de hole burning spectral. La technique d'absorption saturée consiste à analyser, par transmission d'un faisceau monochromatique accordable, ce "trou » de largeur $2 \times \Gamma_{\text {hom }}$ creusé dans le profil de la bande d'absorption. D'abord appliquées aux vapeurs atomiques, où l'élargissement inhomogène est produit par l'effet Doppler [4], ces méthodes spectroscopiques se sont ensuite étendues aux solides [5-7].

La figure 3 présente un exemple de résultats obtenus par spectroscopie de saturation, sur la transition $S_{1}-S_{0}$ 0-0 d'un colorant organique, la porphyrine. Il $s$ 'agit d'une étude de la variation de $\Gamma_{\text {hom }}$ en fonction de la température $T$, la porphyrine étant insérée dans différents matériaux [8]. L'élargissement en fonction de $\mathrm{T}$ dépend beaucoup de la nature de matrice hôte. À $1 \mathrm{~K}$, la largeur homogène ne dépasse pas $10 \mathrm{MHz}$ dans un cristal de $\mathrm{n}$-decane mais atteint $600 \mathrm{MHz}$ dans une matrice amorphe de méthyltétrahydrofurane. Lorsque la température tend vers zéro, toutes les courbes convergent vers la largeur naturelle de la raie, égale à la moitié de l'inverse de la durée de vie du niveau supérieur de la transition. Il est remarquable que la contribution de la matrice à l'élargissement homogène disparaisse à $T=0$, alors même que subsiste le déplacement inhomogène de la fréquence de transition. Ceci reflète les caractères dynamique de l'un et statique de l'autre.

Lorsque le centre absorbant est porté dans un état excité, sa distribution électronique se modifie. La matrice hôte doit se déformer pour s'ajuster à cette transformation et atteindre un nouvel état d'équilibre. Le couplage entre la transition électronique et le réarrangement de la matrice est connue sous le nom de couplage électron-phonon. En raison de ce phénomène, la composante purement électronique de la raie homogène, dite raie à zéro phonon, de largeur $\Gamma_{\text {hom }}$, est flanquée sur son aile bleue d'une bande latérale de phonon, beaucoup plus large. L'élévation de la température a pour effet non seulement d'élargir la raie à zéro phonon mais surtout de l'effacer rapidement au profit de la bande latérale de phonon.

En permettant de modifier localement le profil d'absorption, le hole burning spectral apparaît comme un mécanisme potentiel d'enregistrement sélectif en fréquence. Cependant, lorsque les centres actifs se comportent comme de simples systèmes à deux niveaux d'énergie, ils reviennent rapidement à leur état fondamental et le trou spectral s'efface. En revanche des canaux de phototransformation peuvent s'ouvrir dans le niveau excité de centres actifs plus complexes. Alors l'état final du système differe de son état fondamental initial et le trou creusé dans le profil de la bande d'absorption peut survivre pendant des heures, voire des jours entiers, à basse température [7]. Nous désignerons dans la suite sous le sigle PSHB (persistent spectral hole burning) les matériaux qui possèdent cette propriété.

La bande d'absorption se comporte comme une véritable plaque photographique dans l'espace des fréquences. La largeur inhomogène $\Gamma_{\text {inh }}$ représente la dimension de la plaque tandis que le "grain de l'émulsion » est donné par $\Gamma_{\text {hom }}$. Deux grandes classes de matériaux ont été explorées, qui correspondent à des valeurs très différentes des paramètres $\Gamma_{\text {inh }}$ et $\Gamma_{\text {homm }}$. Dans le cas des molécules organiques dispersées dans des verres ou des polymères, la largeur inhomogène atteint plusieurs $\mathrm{THz}$ tandis que la largeur homogène varie entre quelques dizaines de $\mathrm{MHz}$ et quelques $\mathrm{GHz}$ à la température de l'hélium liquide. Le nombres de points 
séparables à l'intérieur du profil d'absorption varie donc entre $10^{3}$ et $10^{5}$. Dans le cas des ions de terres rares insérés dans des cristaux inorganiques, la largeur inhomogène varie entre quelques $\mathrm{GHz}$ et quelques centaines de $\mathrm{GHz}$ suivant la régularité du réseau cristallin. La largeur homogène est typiquement de l'ordre de $100 \mathrm{KHz}$ à $5 \mathrm{~K}$, mais peut descendre jusqu'à $100 \mathrm{~Hz}$ à $1.4 \mathrm{~K}$ [9].

La figure 4 illustre quelques mécanismes susceptibles de maintenir la gravure au delà de la durée de vie radiative du niveau supérieur de la transition optique. Le premier représente une transformation photochimique dans la chlorine, un dérivé de la porphyrine. Lorsque la molécule est portée dans le premier état électronique excité par absorption à $634 \mathrm{~nm}$, les deux protons au centre du cycle polypyrrolique peuvent subir une rotation de $90^{\circ}$ qui déplace la fréquence de transition à $578 \mathrm{~nm}$. Lors du retour à l'état fondamental, le déficit d'absorption à la fréquence d'excitation est alors préservé. La transformation chimique est irréversible, tout au moins tant que le matériau est maintenu à basse température. Cependant le rendement quantique -probabilité de réaction par photon absorbé- est très faible, de l'ordre de $10^{-3}$, et la molécule excitée revient préférentiellement vers son état initial sans subir de transformation. Un autre facteur contribue à ralentir la gravure. Au lieu de revenir directement à l'état fondamental, la molécule relaxe, avec une probabilité de l'ordre de $90 \%$, vers le niveau triplet de l'état électronique excité. D'une durée de vie de plusieurs dizaines de $\mathrm{ms}$, ce triplet constitue un goulot d'étranglement dans le processus de pompage optique et empêche la molécule d'être rapidement disponible pour un nouveau cycle d'excitation. En conclusion, la probabilité de phototransformation d'une molécule ne dépasse pas quelques $10^{-2} \mathrm{~s}^{-1}$, quelle que soit l'énergie lumineuse disponible. Un mécanisme plus efficace serait une phototransformation à deux photons de couleurs différentes qui, outre qu'elle supprimerait les temps morts, assurerait aussi la fixation de la gravure [10].
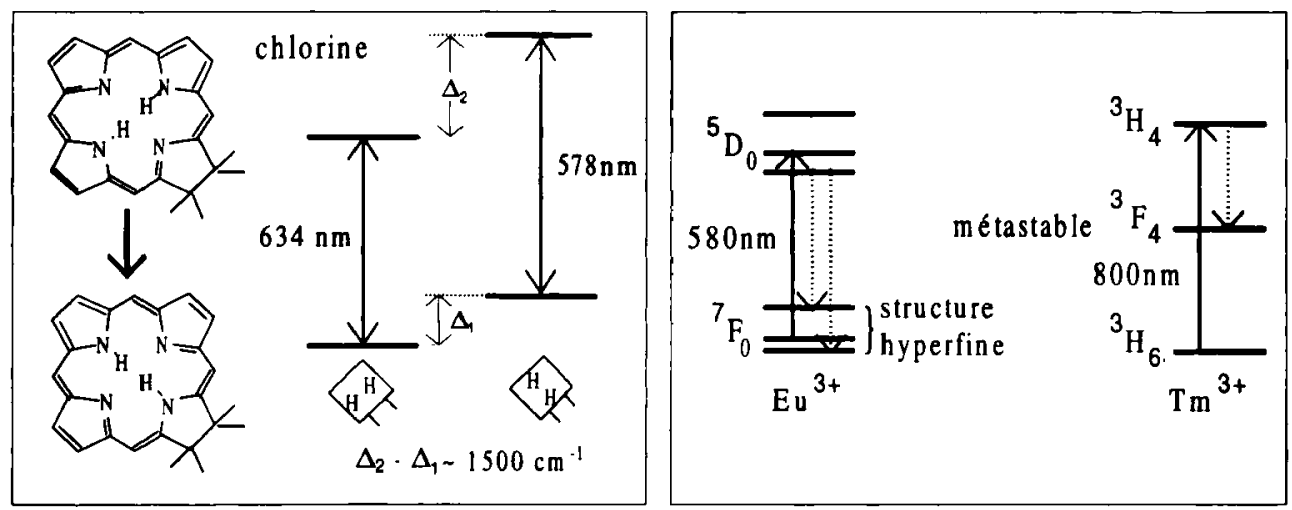

Figure 4 : Exemples de mécanismes de maintien du « hole burning » spectral dans les matériaux organiques et les ions de terres rares.

Dans les ions de terres rares d'autres processus permettent de sauvegarder la gravure spectrale effectuée par « hole burning ». Ainsi, dans l'ion Europium $\mathrm{Eu}^{3+}$ [11], l'excitation sur la transition ${ }^{7} \mathrm{~F}_{0^{-}}{ }^{5} \mathrm{D}_{0}$ modifie par pompage optique la répartition ionique entre les sous-niveaux hyperfins du fondamental ${ }^{7} \mathrm{~F}_{0}$. Le retour à l'équilibre thermique de cette distribution demande plusieurs heures pendant lesquelles la gravure est préservée. La largeur inhomogène disponible est limitée à quelques $\mathrm{GHz}$ à cause du chevauchement des raies associées aux différents sous-niveaux hyperfins. On peut aussi tirer parti de la présence d'un niveau 
métastable vers lequel relaxe l'ion excité optiquement avant de retourner au fondamental. C'est le cas dans l'ion Thulium $\mathrm{Tm}^{3+}$ [12] où la gravure ne subsiste cependant pas plus d'une dizaine de millisecondes, durée de vie du niveau intermédiaire ${ }^{3} F_{4}$. Avec une largeur homogène de quelques $\mathrm{kHz}$ seulement à $1.5 \mathrm{~K}$ on peut espérer distinguer jusqu'à $10^{7}$ canaux spectraux sur une largeur inhomogène de l'ordre de $10 \mathrm{GHz}$ comme celle qu'on rencontre dans $\mathrm{Tm}^{3+}$ :YAG. Des recherches très récentes font apparaître une bande d'absorption de $270 \mathrm{GHz}$ dans $\mathrm{Tm}^{3+}$ :YLuAG, dont la matrice cristalline est un peu plus désordonnée que le YAG. Le nombre de canaux spectraux accessibles atteint alors $10^{9}$ à $1.5 \mathrm{~K}$. La grande finesse de la raie homogène se paie par une très faible force d'oscillateur, de l'ordre de $10^{-8}$, qui contraint à mettre en oeuvre des flux lumineux élevés. Dans le cas précis d'un cristal dopé 0.1 at $\% \mathrm{Eu}^{3+}: \mathrm{Y}_{2} \mathrm{SiO}_{5}$ l'exposition requise pour creuser jusqu'à saturation un trou monochromatique est de $8 \mathrm{~mJ} / \mathrm{cm}^{2}$ environ [13].

La gravure n'implique pas seulement des mécanismes internes aux centres absorbants. Ainsi a-t-on creusé des trous spectraux de plusieurs heures de durée de vie dans la bande d'absorption à $793 \mathrm{~nm}$ de l'ion $\mathrm{Tm}^{3+}$, lorsque celui-ci est inséré dans une matrice de $\mathrm{CaF}_{2}: \mathrm{D}^{-}$. La migration d'ions $\mathrm{D}^{-}$vibrationnellement excités par transfert d'énergie explique vraisemblablement cet effet [14] (figure 5). La largeur d'un trou atteint $20 \mathrm{MHz}$ environ, pour une largeur inhomogène de $20 \mathrm{GHz}$.

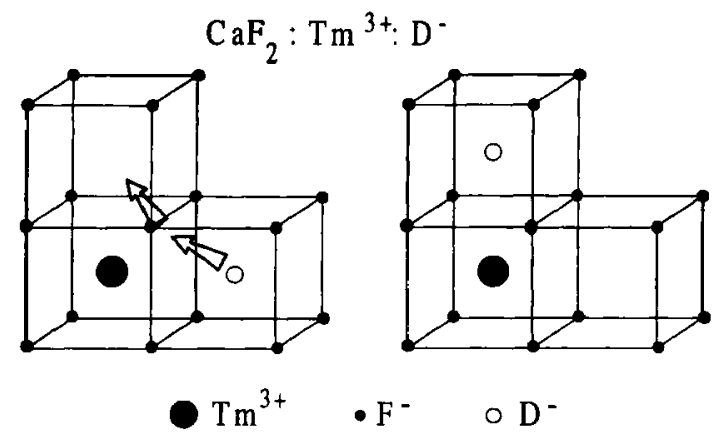

Figure 5 : Mécanisme probable de gravure dans $\mathrm{CaF}_{2}: \mathrm{Tm}^{3+}: \mathrm{D}^{-}$. Dans le cristal excité sur la transition à $793 \mathrm{~nm}$ du thulium, l'ion $\mathrm{D}^{-}$quitte un site pour migrer vers une autre position stable.

\section{HOLOGRAPHIE SPATIALE SÉLECTIVE EN LONGUEUR D'ONDE}

Dans le paragraphe précédent nous avons examiné le mécanisme physique d'enregistrement sélectif en longueur d'onde en laissant de côté les dimensions spatiales du matériau utilisé. En fait le volume spatial de mémoire reste tout entier disponible à chaque longueur d'onde. Dans la perpective du stockage optique à grande capacité, des démonstrations de mémoires de pages à trois dimensions ont été réalisées [13, 15]. Les informations contenues dans une page spatiale à deux dimensions sont gravées ou lues en parallèle. Chaque page est caractérisée par une adresse spectrale qui constitue la troisième dimension.

La technique d'enregistrement et de lecture, représentée sur la figure 6, ne se distingue de l'holographie conventionnelle que par l'utilisation d'un laser accordable en fréquence qui permet d'accéder séquentiellement aux différentes adresses spectrales. La profondeur du trou spectral creusé par le laser monochromatique dépend de l'énergie reçue. Comme la 
distribution spatiale de l'énergie reçue est déterminée par la figure d'interférence des faisceaux objet et référence, un hologramme d'absorption s'inscrit dans le matériau photosensible suivant la distribution spatiale du « hole burning » spectral. Le hologramme existe seulement à la longueur d'onde de la lumière de gravure. Quand un laser sonde est accordé à cette longueur d'onde, son faisceau est diffracté. Il ne rencontre plus l'enregistrement dès que sa fréquence s'écarte de plus de $2 \Gamma_{\text {hom }}$ de celle du laser de gravure. La sélectivité du « hole burning » est locale par essence et n'a aucun rapport avec la sélectivité de Bragg conventionnelle, qui dépend de l'épaisseur de l'échantillon. Chaque réseau gravé par «hole burning" spectral n'occupe donc qu'un canal de largeur $2 \Gamma_{\text {hom }}$ dans l'espace des fréquences. Au sein d'un matériau de largeur inhomogène $\Gamma_{\text {inn }}$, peuvent cohabiter $\Gamma_{\text {inh }} /\left(2 \Gamma_{\text {nom }}\right)$ hologrammes indépendants. Chacun se construit sur un groupe de centres actifs spécifique et, dans un matériau parfait tout au moins, l'efficacité de diffraction de chacun d'eux est indépendante de leur nombre. Elle peut atteindre quelques pour cent. Ce comportement, caractérisique d'un enregistrement multicanal, diffère de celui du multiplexage en fréquence dans un photoréfractif. Dans ce dernier cas en effet, les réseaux gravés à différentes longueurs se partagent l'efficacité de diffraction maximum disponible. L'efficacité de diffraction sur chaque hologramme décrô̂t alors comme l'inverse du carré du nombre de hologrammes multiplexés.

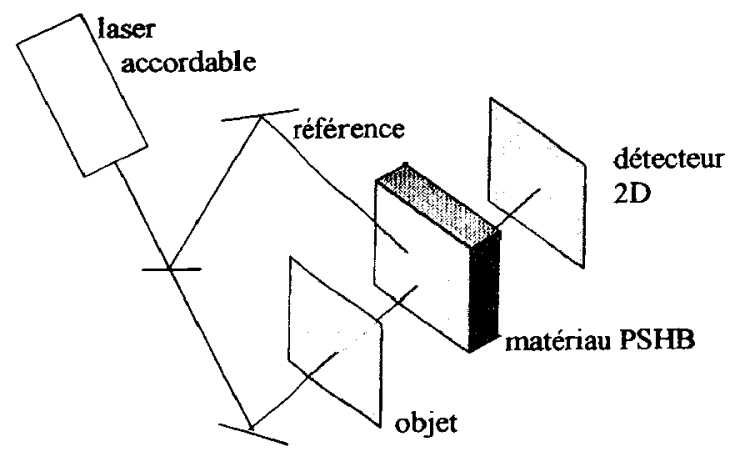

Figure 6 : Schéma de principe d'une mémoire de pages à trois dimensions, une de longueur d'onde et deux d'espace.

La première étude a été réalisée à ETH Zürich par le groupe de U. Wild dans un matériau organique, la chlorine, dispersée dans un film de polyvinylbutyral de $65 \mu \mathrm{m}$ d'épaisseur, sur une surface de un pouce carré. Dans cette mince feuille ont été stockées 6000 pages de $20 \times 20$ pixels, réparties sur les $4 \mathrm{THz}$ de largeur de la bande d'absorption à $634 \mathrm{~nm}$ [15]. La vitesse d'accès aux adresses spectrales reste très faible en raison des difficultés rencontrées pour balayer un laser monochromatique sur un aussi grand intervalle. Par ailleurs la faible efficacité quantique du processus photochimique de gravure requiert des expositions de longue durée. Même réduite à 4 secondes par page, la durée cumulée des 6000 expositions atteint plus de 6 heures! Sur la transition ${ }^{7} \mathrm{~F}_{0}{ }^{-} \mathrm{D}_{0}$ à $580 \mathrm{~nm}$ dans l'ion de terre rare $\mathrm{Eu}^{3+}$ inséré dans un cristal de $\mathrm{Y}_{2} \mathrm{SiO}_{5}$ l'efficacité quantique de photo-transformation est environ $10^{3}$ fois plus grande, la largeur inhomogène de la bande d'absorption $10^{3}$ fois plus petite pour un nombre de canaux spectraux au moins 10 fois plus élevé que dans l'échantillon organique précédent. L'enregistrement et la lecture cinématographiques en temps réel ont pu être démontrés dans 
un tel matériau à l'occasion d'une collaboration entre des groupes de NTT et Olympus Co.[13]. Cependant la très grande stabilité du laser $\left(10^{-12}\right)$ et la basse température $(7 \mathrm{~K})$ restent prohibitives pour un domaine d'application où des techniques beaucoup moins complexes et coûteuses continuent à progresser.

Se tournant vers des applications plus spécialisées, on pourrait exploiter l'association des coordonnées de fréquence et d'espace, afin de réaliser par exemple des opérations de balayage angulaire rapide, d'analyse spectrale, de corrélation. Des architectures à base de dispositifs acousto-optiques possèdent ces fonctionnalités mais les performances des matériaux PSHB permettent de concevoir des processeurs optiques dotés d'une bande passante 10 à 50 fois plus grande et d'un produit temps $\times$ fréquence considérable. L'exemple de l'analyse spectrale à large bande instantanée et haute résolution [16] est schématisé sur la figure 7 . Il consiste à séparer spatialement les composantes spectrales d'un faisceau lumineux modulé par un signal radiofréquence. Pour ce faire on tire parti de la possibilité de graver, dans un même cristal PSHB, $\Gamma_{\text {inh }} /\left(2 \Gamma_{\text {hom }}\right)$ réseaux de Bragg indépendants. Chaque réseau enregistré à la fréquence $v^{(n)}$ est caractérisé par un vecteur spécifique $\mathbf{k}_{2}{ }^{(n)}-\mathbf{k}_{1}{ }^{(\mathbf{n})}$ défini par les vecteurs d'onde $\mathbf{k}_{\mathbf{1}}{ }^{(\mathrm{n})}$ et $\mathbf{k}_{2}{ }^{(n)}$ des deux faisceaux de gravure. Supposons que tous les vecteurs d'onde $\mathbf{k}_{1}{ }^{(\mathbf{n})}$ soient orientés dans la direction commune $\mathbf{k}_{\mathrm{l}}$. Alors les différentes composantes spectrales $v^{(\mathrm{n})} \mathrm{d}^{\prime} \mathbf{u n}$ faisceau polychromatique incident suivant $\mathbf{k}_{1}$ seront séparées et diffractées dans les directions $\mathbf{k}_{2}{ }^{(n)}$. Elles pourront être détectées simultanément sur une matrice de photodétecteurs. Il faut souligner encore une fois que le filtrage spectral, dans un matériau PSHB, n'est pas lié à la sélectivité de Bragg mais au caractère résonnant de l'absorption à l'échelle atomique. À titre d'illustration, fixons comme objectif une résolution de $10 \mathrm{MHz}$, aisément accessible dans un matériau composé d'ions de terre rare. Pour atteindre la même résolution par sélectivité de Bragg, un cristal de $\mathrm{LiNbO}_{3}$ de 6 mètres de long, utilisé en configuration de contrepropagation, serait nécessaire.
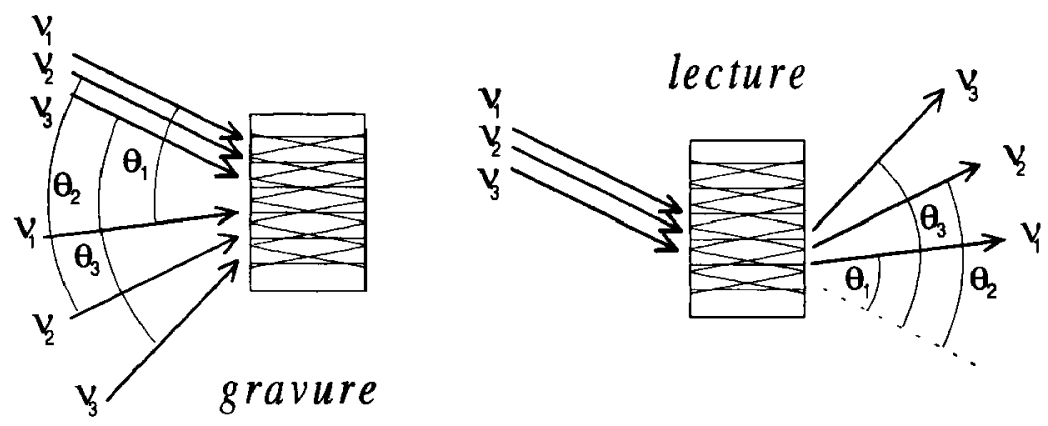

Figure 7 : Principe de la séparation angulaire des fréquences temporelles. Dans un premier temps les réseaux de Bragg monochromatiques sont gravés sous différentes incidences. Ils sont alors disponibles pour diffracter différentes fréquences dans différentes directions.

La dimension temporelle est pour l'instant absente de cet exposé. Pourtant fréquence et temps, variables conjuguées, sont étroitement liées et nous n'avons pu ignorer la dimension temporelle qu'en supposant implicitement que les durées mises en jeu pour la gravure, le balayage de fréquence ou la lecture, étaient toutes très supérieures à l'inverse de la largeur homogène. Nous montrons dans le paragraphe suivant qu'il est possible de faire l'économie de cette hypothèse. 


\section{ASPECTS DYNAMIQUES}

Par la méthode d'adressage en longueur d'onde décrite dans le paragraphe précédent, on accède successivement aux différents canaux spectraux définis dans la bande d'absorption du matériau mémoire. Le balayage séquentiel de ces adresses est représenté sur la figure 8 . Si le temps passé à chaque adresse est noté $\tau$, alors une adresse occupe un intervalle spectral au moins égal à $\tau^{-1}$. La vitesse de transfert des données, égale elle aussi à $\tau^{-1}$, semble entrer en conflit avec la capacité de mémoire. En effet, la séparation des adresses spectrales, réparties sur l'intervalle $\Gamma_{\text {inh }}$, exige que le produit du nombre d'adresses par la vitesse de transfert soit inférieur à $\Gamma_{\mathrm{inh}}$. Cette observation, si elle était correcte, conduirait à une vitesse d'accès maximale $\Gamma_{\text {hom }}^{-1}$ dans une mémoire dont on exploiterait la pleine capacité $\Gamma_{\text {inh }} / \Gamma_{\text {hom }}$. Nous allons montrer cependant que les enregistrements peuvent se chevaucher dans l'espace des fréquences sans que cela nuise à leur lisibilité. C'est une propriété de multiplexage analogue à celle qu'on rencontre en holographie spatiale conventionnelle. Elle ne doit pas surprendre. Tout comme en holographie spatiale on enregistre la distribution spatiale du champ lui-même et non celle de son intensité, c'est bien la distribution spectrale du champ qu'on enregistre dans le matériau PSHB.

Lorsque les champs de référence et de lecture sont deux ondes planes de même direction de propagation et de durée inférieure à $\Gamma_{\text {inh }}{ }^{-1}$, leur spectre est plat sur l'intervalle de gravure et le produit de leurs amplitudes spectrales prend la forme :

$$
\tilde{\mathrm{b}} *(\mathbf{r}, v) \widetilde{\mathrm{c}}(\mathbf{r}, v)=\exp -2 \mathrm{i} \pi v \mathrm{t}_{23}
$$

où $t_{23}$ désigne la séparation temporelle entre les deux impulsions. D'après les équations $1-4$, $s(r, t)$ reproduit alors la forme de l'objet $a(r, t)$, à la translation de $t_{23}$ près. Le hologramme $\tilde{a}(\mathbf{r}, v) \widetilde{b}^{*}(\mathbf{r}, v)$ associé à cet objet s'étend sur un intervalle spectral au plus égal à $\Gamma_{\text {inh }}$, sur lequel il est enregistré avec la résolution $\Gamma_{\text {hom }}$. Cela correspond dans le domaine temporel, d'après les propriétés de la transformation de Fourier et compte tenu de la brièveté de l'impulsion référence, à un objet de durée maximale $\Gamma_{\text {hom }}{ }^{-1}$ dont la structure est sauvegardée avec la résolution $\Gamma_{\mathrm{inh}}{ }^{-1}$. Un train de $\Gamma_{\mathrm{inh}} / \Gamma_{\text {hom }}$ impulsions de durée $\Gamma_{\mathrm{inh}}{ }^{-1}$ satisfait ces conditions et représente bien une capacité maximale de $\Gamma_{\text {inh }} / \Gamma_{\text {hom }}$ adresses explorées à la vitesse maximale $\Gamma_{\mathrm{inh}}$, vitesse $\Gamma_{\mathrm{inh}} / \Gamma_{\text {hom }}$ fois plus élevée que ne le permettraient des adresses spectrales séparées.

Une telle cadence de transfert dépasse cependant les performances de la plupart des dispositifs électroniques et on cherche plutôt à obtenir une vitesse intermédiaire entre $\Gamma_{\text {inh }}$ et $\Gamma_{\text {hom }}$. L'holographie spectro-temporelle permet d'atteindre cet objectif. En effet, il n'est pas nécessaire d'utiliser des impulsions courtes pour exploiter la pleine capacité de la mémoire, tout en atteignant une vitesse très supérieure à $\Gamma_{\text {hom }}$. On peut restituer un champ objet spatiotemporel $a(r, t)$ si les deux conditions suivantes sont vérifiées :

- le produit $\widetilde{a}(\mathbf{r}, v) \tilde{b}^{*}(\mathbf{r}, v)$ s'étend sur un intervalle inférieur à $\Gamma_{\text {inh }}$ et il ne présente pas de structure plus étroite que $\Gamma_{\text {hom }}$.

- le produit des spectres référence et lecture se réduit à $\tilde{b} *(\mathbf{r}, v) \tilde{c}(r, v)=\exp -2 i \pi v t_{23}$ sur l'intervalle de gravure. 


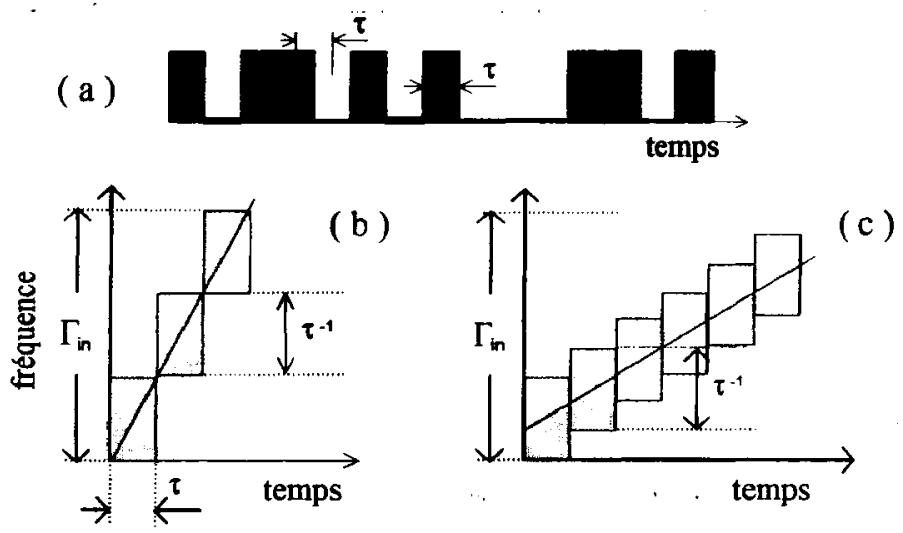

Figure 8 : Les données atteignent ou quittent la mémoire à la vitesse $\tau^{-1}$ (a). Leur séparation spectrale limite le nombre des adresses à $\Gamma_{\text {inh }} \tau$ (b). Par multiplexage on atteint pourtant la capacité d'adresses maximale $\Gamma_{\text {inh }} / \Gamma_{\text {hom }}$ à la vitesse $\Gamma_{\text {inh }}(c)$.

Sans faire appel à des impulsions brèves, on peut satisfaire ces conditions à l'aide de champs à glissement linéaire de fréquence de la forme exp $2 \mathrm{i} \pi\left(v_{0}+\alpha t\right)$ t. Les données sont alors gravées et lues sur un intervalle spectral de largeur $\alpha \mathrm{T} / 2$ pendant la durée $\mathrm{T}$ du balayage. Par cette méthode ont été stockés expérimentalement 1760 bits sur un intervalle de $900 \mathrm{MHz}$ [17] dans un échantillon de $\mathrm{Tm}^{3+}$ :YAG. La séparation spectrale des données aurait limité la vitesse de transfert à $900 / 1760 \approx 0.5 \mathrm{Mbits} / \mathrm{s}$. Le multiplexage spectral a permis d'atteindre une vitesse de $20 \mathrm{Mbits} / \mathrm{s}$. Pour cela on a dû multiplexer environ 40 adresses spectrales en chaque point du profil d'absorption, suivant le schéma de la figure $8 \mathrm{c}$.

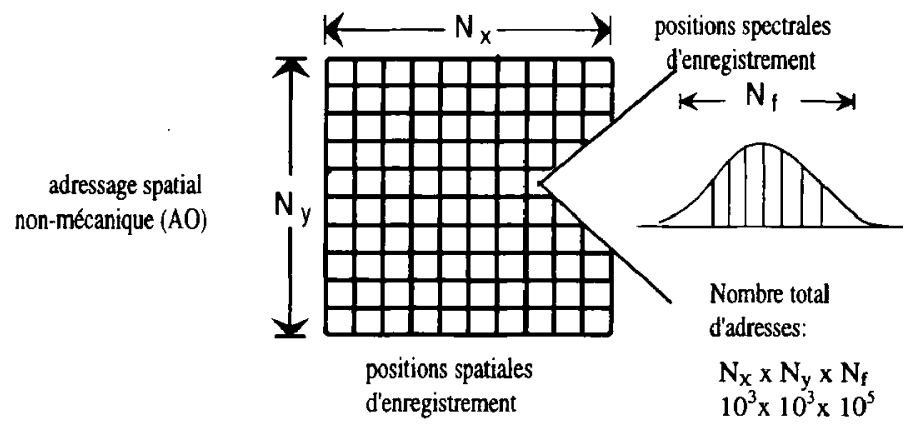

Figure 9 : Schéma de principe d'une mémoire à trois dimensions à accès séquentiel rapide (ODRAM). Chaque position spatiale est occupée par $10^{5}$ adresses spectrales. Ce groupe d'adresses serait parcouru à une vitesse comprise entre 10 et $1000 \mathrm{Mbits} / \mathrm{s}$. Les $10^{3} \times 10^{3}$ positions spatiales, réunies sur une petite surface d'environ $7 \mathrm{~cm}^{2}$, pourraient être explorées à l'aide d'un déflecteur acousto-optique.

T. Mossberg, qui a réalisé ce travail à l'Université d'Oregon à Eugene insiste sur deux autres propriétés de ce type d'enregistrement. D'une part, en ajoutant la dimension spectrale au volume de mémoire on atteint des densités spatiales élevées : $1.2 \mathrm{Gbits} / \mathrm{cm}^{2}$ dans cet exemple, alors qu'un dixième seulement de la bande d'absorption est occupée. D'autre part on accède à un si grand nombre de données en éclairant un seul point de l'échantillon, sans 
déplacement mécanique, que la dimension spectrale peut constituer à elle seule un axe « rapide» de grande capacité. Mossberg tient le raisonnement suivant. Compte tenu des limites imposées par le bruit, il faut environ $n=10^{4}$ atomes pour enregistrer 1 bit. Par ailleurs l'intensité du signal est optimale dans un échantillon de densité optique voisine de 1 . Il en résulte une valeur maximale de la densité surfacique des données enregistrées. Dans une mémoire orientée vers le stockage d'images bidimensionnelles de haute définition, on s'accommode de cette contrainte en élargissant le spectre du laser. On atteint ainsi davantage de centres actifs à travers un élément de surface et on réduit de ce fait l'aire requise pour produire le signal minimum détectable. L'amélioration de la résolution spatiale se paie par une dégradation de la résolution spectrale. Les centres actifs disponibles se partagent entre des canaux spectraux moins nombreux. Au contraire, et c'est l'objectif visé par Mossberg, on peut

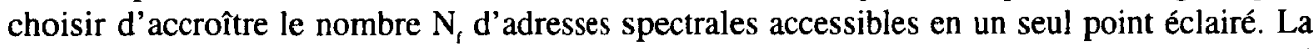
surface à réserver à chaque pile d'adresses spectrales est alors déterminée par le nombre total $\mathrm{nN}_{\mathrm{f}}$ d'atomes requis, divisé par la densité surfacique des atomes actifs dans une lame de densité 1 [18]. Pour $\mathrm{N}_{\mathrm{f}}=10^{5}$ dans $\mathrm{Tm}^{3+}$ :YAG la surface nécessaire pour chaque pile est environ $400 \mu^{2}$. Sur une plaque de 1 pouce carré on peut rassembler $10^{6}$ piles spectrales, adressables en $10 \mu \mathrm{s}$ environ par déflecteur acousto-optique, et réaliser ainsi une unité de mémoire de 10 gigaoctets, avec une vitesse de transfert de quelques dizaines de $\mathrm{Mbits} / \mathrm{s}$ à l'intérieur de chaque pile de $10^{5}$ adresses, et un temps de latence de $10 \mu \mathrm{s}$ pour la commutation entre les points. Une telle mémoire, dont le principe est schématisé sur la figure 9, offrirait la capacité d'un support magnétique avec une vitesse d'accès beaucoup plus élevée. S'appuyant sur ce schéma, un prototype de mémoire séquentielle rapide est en cours de réalisation dans le cadre de la «startup company» TEMPLEX à Eugene.

En examinant le problème de la vitesse de transfert dans une mémoire sélective en fréquence nous avons rencontré des propriétés liées à la cohérence dans le domaine spectrotemporel. Le parallèle entre espace et temps/fréquence se poursuit dans le paragraphe suivant. Des architectures familières en holographie spatiale sont adaptées à la conception de processeurs optiques spectro-temporels.

\section{PROCESSEURS SPECTRO-TEMPORELS}

Dans le paragraphe 5 nous avons considéré la situation où la référence d'écriture $b(r, t)$ et l'onde de lecture $c(r, t)$ sont des impulsions brèves qui se propagent dans la même direction. Le signal se réduit alors à $\mathrm{a}\left(\mathbf{r}, \mathrm{t}-\mathrm{t}_{23}\right)$ et reproduit fidèlement le profil temporel du champ objet enregistré. C'est la configuration choisie pour réaliser une mémoire. Plus généralement l'équation 3 présente une forme similaire à celle qui, en holographie de Fourier, constitue la base de la conjugaison de phase ou de l'intercorrélation d'images [19]. La coordonnée temporelle y joue le rôle tenu par les coordonnées d'espace en holographie conventionnelle. La situation équivalente à celle de la conjugaison de phase se manifeste lorsque $a(r, t)$ et $c(r, t)$ sont des impulsions brèves. Dans le domaine temporel, l'hologramme réalise alors une opération de renversement du temps, construisant $b^{*}(r,-t)$ à partir du champ enregistré $b(r, t)$. À la correction de distorsion par conjugaison de phase répond, dans le domaine spectrotemporel, la correction de dispersion par inversion du temps [20]. L'opération d'intercorrélation est effectuée lorsque $a(r, t)$ est une impulsion brève. L'équation 3 se réduit alors à l'expression :

$$
s(\mathbf{r}, \mathbf{t})=b(\mathbf{r}, \mathbf{t}) * c(\mathbf{r}, \mathbf{t})
$$


qui fait apparaître le signal comme la corrélation entre la forme temporelle $c(r, t)$, présentée à l'entrée du corrélateur, et la référence $b(r, t)$, enregistrée dans la mémoire PSHB.

Le matériau PSHB travaillant comme un processeur optique se distingue par une bande passante instantanée qui, dans les ions de terres rares, dépasse $100 \mathrm{GHz}$ avec un produit temps $\times$ bande passante supérieur à $10^{6}$ et, dans les matériaux organiques, dépasse $4 \mathrm{THz}$ avec un produit temps $\times$ bande passante de l'ordre de $10^{4}$. En outre les dimensions spatiales du processeur restent disponibles, par exemple pour combiner la sélectivité angulaire au traitement temporel.
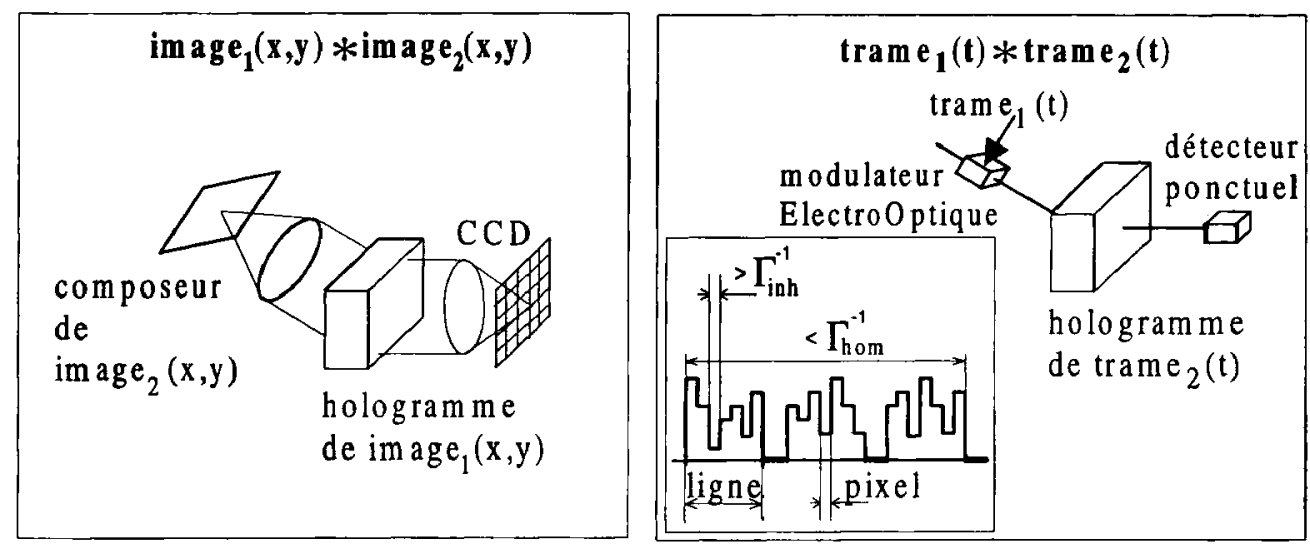

Figure 10 : Principe du corrélateur de trames. À gauche, corrélateur spatial conventionnel : la transformée de Fourier de l'image d'entrée bidimensionnelle image ${ }_{2}(x, y)$ est diffractée sur le hologramme de Fourier de l'image de référence; le signal de corrélation est détecté sur une matrice $\mathrm{CCD}$. À droite la porteuse optique, modulée par

la trame d'entrée trame $e_{1}(t)$, est diffractée sur le hologramme spectral de la trame de référence. Le signal de corrélation est analysé dans le temps à l'aide d'un détecteur ponctuel rapide. La durée de chaque trame est limitée à $\Gamma_{\text {hom }}{ }^{-1}$ et la durée d'un pixel est au moins égale à $\Gamma_{\text {inh }}{ }^{-1}$

Proposée par Kelvin Wagner de l'Université du Colorado [21] dans le domaine de la corrélation d'images, une architecture met ces performances en évidence. Le principe en est présenté sur la figure 10. Dans un corrélateur optique conventionnel il est en général nécessaire de convertir un signal vidéo en image bidimensionnelle avant de l'adresser à un composeur d'image puis de convertir à nouveau en signal vidéo l'image de corrélation recueillie sur un détecteur bidimensionnel. Un corrélateur PSHB fait l'économie de ces deux opérations. La trame vidéo de l'image d'entrée reste sous la forme d'une séquence d'impulsions. Transformée en train d'impulsions lumineuses, elle est corrélée à la forme de référence enregistrée dans le matériau PSHB. Le signal de corrélation apparaît lui-même comme une séquence d'impulsions que reçoit un détecteur ponctuel. Compte tenu des caractéristiques des matériaux PSHB, un tel dispositif ne peut pas traiter les signaux vidéo standard basse fréquence. En revanche il pourrait faire merveille à des cadences d'analyse très élevées de plusieurs milliers d'images par seconde. Par ailleurs, plusieurs signaux de corrélation, correspondant à des formes de référence différentes, pourraient être détectés simultanément sur différents détecteurs ponctuels puisque les propriétés de parallélisme spatial restent disponibles. 
Lorsque le matériau actif est composé d'ions de terre rare, les bandes passantes et les temps caractéristiques restent compatibles avec les performances des modulateurs acoustooptiques ou électro-optiques et des photodétecteurs disponibles [22]. Il en va différemment dans le domaine des bandes passantes de l'ordre du $\mathrm{THz}$, caractéristique des colorants organiques dispersés dans des polymères. Ces très hautes fréquences restent hors d'atteinte des dispositifs électroniques. Pour réaliser une démonstration expérimentale de reconnaissance de forme temporelle par corrélation dans ce domaine de fréquences nous avons été confrontés au triple défi d'inscrire une « forme » cohérente de plusieurs $\mathrm{THz}$ de largeur dans le spectre d'une impulsion lumineuse, de graver cette forme sans distorsion dans le matériau sélectif en fréquence, puis de détecter le pic d'intercorrélation avec une résolution subpicoseconde.

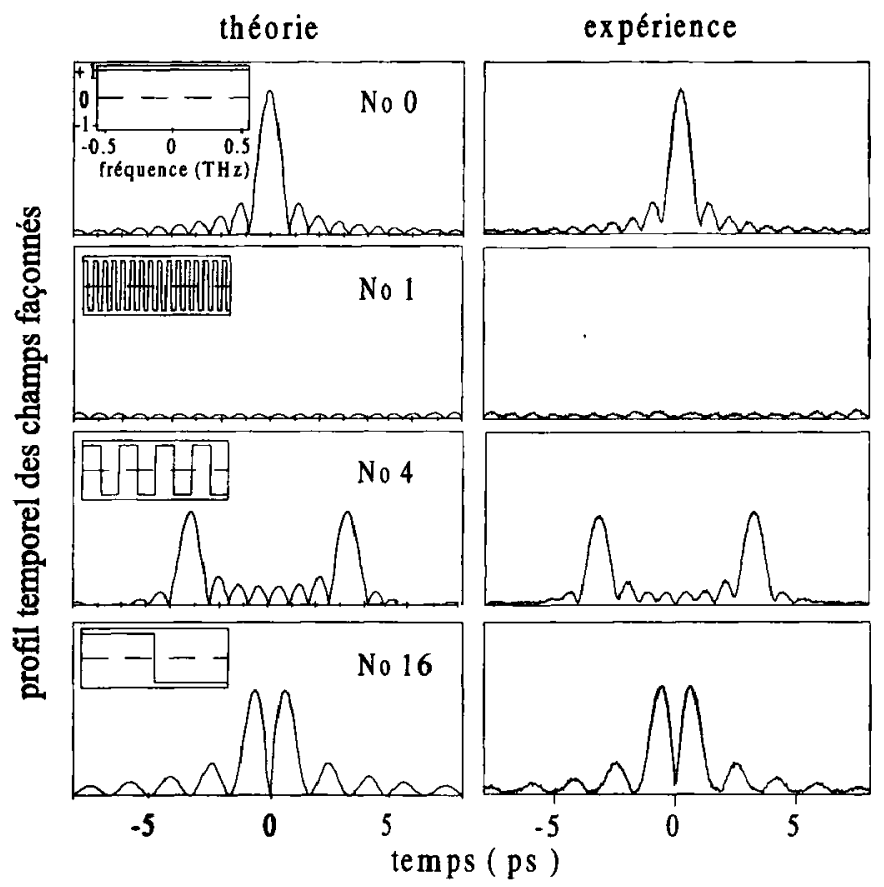

Figure 11 : Profils temporels, théoriques et expérimentaux, du champ façonné spectralement. Les numéros repèrent les formes spectrales (représentées en insert) choisies dans la famille des 32 fonctions orthogonales.

Dans notre démonstration, nous définissons une famille de 32 facteurs de phase spectraux orthogonaux qui constituent les formes à reconnaître. L'une quelconque de ces formes est enregistrée par holographie spectro-temporelle dans le matériau mémoire. Les 32 formes sont ensuite successivement inscrites sur des impulsions tests qui sont dirigées sur l'échantillon. Le signal diffracté doit présenter un pic d'intercorrélation lorsque la forme spectro-temporelle dont il est chargé coïncide avec le motif de référence enregistré dans la mémoire optique [23]. La figure 11 illustre le réglage final du dispositif de façonnage spectral des impulsions lumineuses et du dispositif de détection interférométrique qui nous donne la résolution subpicoseconde requise. Les graphes de gauche représentent le profil temporel inscrit en théorie sur le spectre des impulsions lumineuses. En insert sont tracées les formes spectrales 
correspondantes. Les graphes de droite représentent ce profil temporel, analysé expérimentalement.

Ce réglage effectué, nous avons pu mener à bien l'expérience de reconnaissance de forme proprement dite. Une forme de référence est d'abord gravée dans le matériau mémoire. Les 32 formes orthogonales sont ensuite successivement inscrites sur le spectre de l'impulsion d'entrée. Pour chacune des impulsions d'entrée ainsi façonnées, on mesure l'amplitude du champ diffracté à l'instant fixe $t=0$, qui correspond à la position du pic d'intercorrélation. Ces séries d'amplitudes dessinent les profils présentés sur la figure 12 . Différentes formes ont été utilisées comme références enregistrées dans le matériau mémoire. Le rapport entre le pic de corrélation vraie, observé Iorsque l'impulsion d'entrée coïncide avec la référence, et le signal de fausse corrélation, obtenu dans le cas contraire, ne semble pas dépendre de la complexité de la forme spectrale enregistrée.

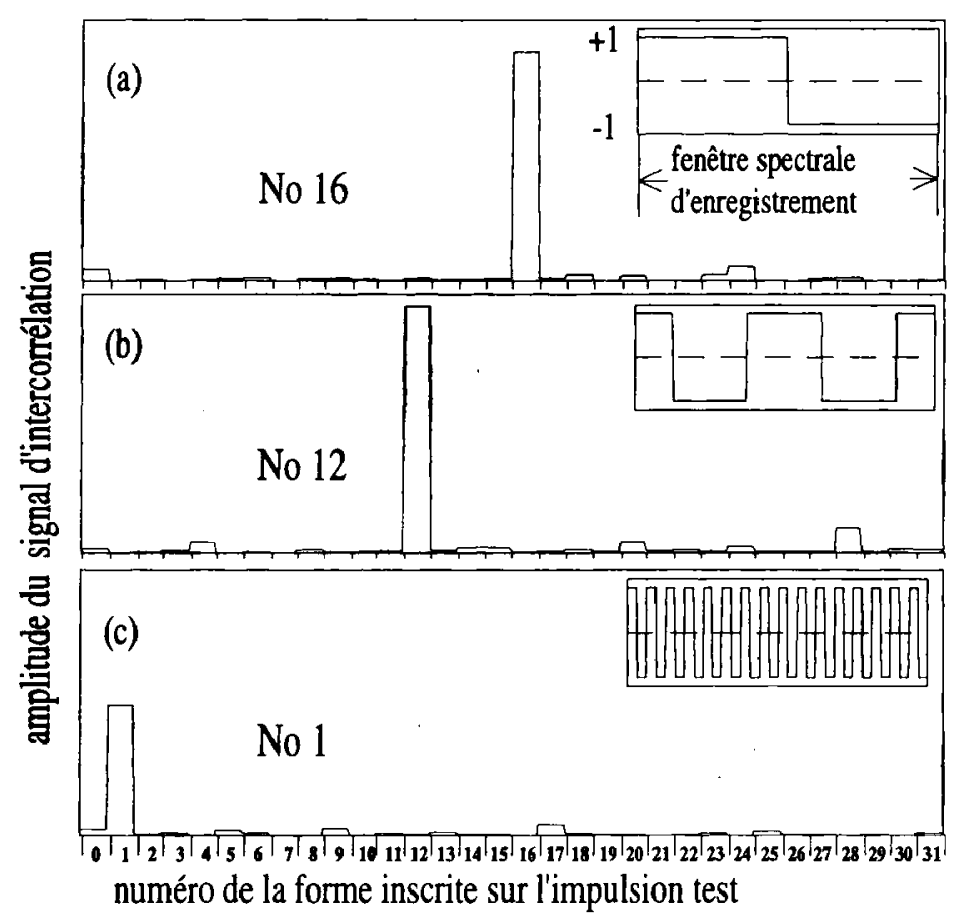

Figure 12 : Dans un premier temps on grave dans le matériau mémoire une forme de référence( $n^{\circ} 1,12$ ou 16 sur la figure). Les 32 formes orthogonales sont ensuite successivement chargées dans le spectre d'une impulsion. La figure représente l'amplitude du signal détecté en réponse à chacune de ces interrogations.

Un corrélateur en matériau PSHB organique pourrait permettre le diagnostic de signaux sub-picoseconde. La procédure de reconnaissance de forme prendrait tout son sens si on pouvait comparer simultanément l'impulsion d'entrée à un ensemble de filtres de référence enregistrés. Ceci est possible à condition d'associer une direction de diffraction spécifique à chaque forme enregistrée, tout comme on associe une direction de diffraction particulière à chaque fréquence dans l'analyseur spectral évoqué dans le paragraphe 4. Le diagnostic d'une forme temporelle serait alors effectué instantanément, et en un seul tir. 


\section{CONCLUSION}

Plutôt qu'une revue des travaux expérimentaux déjà accomplis sur le « hole burning » spectral et ses applications au traitement optique de données, ce bref exposé présente un point de vue peut-être partial sur les difficultés rencontrées et les directions les plus convaincantes aux yeux de l'auteur. L'accent a été mis sur certaines recherches, souvent encore à l'état de projet, mais qui semblent intégrer les promesses de l'enregistrement sélectif en fréquence et les limitations des matériaux employés. Malgré les premiers succès rencontrés dans la réalisation de mémoires optiques rapides, les progrès continus des technologies plus conventionnelles compromettent le passage effectif à l'application. Le traitement optoélectronique du signal ouvre sans doute de meilleures perspectives. Les processeurs potentiels devront probablement tirer le meilleur parti de la combinaison des aspects spatiaux et spectraux des processus mis en jeu. Un fort couplage avec les spécialistes des architectures optoélectroniques semble indispensable.

\section{Références}

[1] Mazurenko Yu.T., Appl. Phys. B 50 (1990) 101-114; Rakuljic G.A., Lerya V. et Yariv A., Opt. Lett. 17 (1992) 1471-73; Rätsep M., Tian M., Grelet F., Le Gouët J.-L., Sigel C. et Roblin M.-L., Opt. Lett. 21 (1996) 1292-1294.

[2] Mossberg T., Opt. Lett. 7 (1982) 77-79.

[3] Fujita M., Nakatsuka H., Nakanishi H. et Matsuoka M., Phys. Rev. Lett. 42 (1979) 974977; Mossberg T.W., Flusberg A., Kachru R. et Hartmann S.R., Phys. Rev. Lett. 42 (1979) 1665-1669; Hesselink W.H. et Wiersma D.A., Phys. Rev. Lett. 43 (1979) 1991-1994.

[4] Hänsch T.W., Shahin I.S. et Schawlow A.L., Phys. Rev. Lett. 27 (1971) 707-710; Nature (Phys. Sci.) London, 235 (1972) 63-65.

[5] Jessop P.E. et Szabo A., Opt. Commun. 33 (1980) 301-302; Jessop P.E., Muramoto T. et Szabo A., Phys. Rev. B 21 (1980) 926-936.

[6] Szabo A., Phys. Rev. B 11 (1975) 4512-4517

[7] Gorkhovskii A.A., Kaarli R.K. et Rebane L.A., JETP Lett. 20 (1974) 216-218; Kharlamov B.M., Personov R.I. et Bykovskaya L.A., Opt. Commun. 12 (1974) 191-193.

[8] Thijssen H.P.H., Van den Berg R.E. et Völker S., Chem. Phys. Lett. 103 (1983) 93-98.

[9] Equall R.W., Sun Y., Cone R.L. et Macfarlane R., Phys. Rev. Lett. 72 (1994) 2179-2182.

[10] Rebane A., Reiss D., Renge I. et Wild Urs P., Chem. Phys. Lett. 262 (1996) 155-160.

[11] Yano R., Mitsunaga M. et Uesugi N., J. Opt. Soc. Am. B 9 (1992) 992-997.

[12] Macfarlane R., Opt. Lett. 18 (1993) 829-31, 1958-1960.

[13] Mitsunaga M., Uesugi N., Sasaki H. et Karaki K., Opt. Lett. 19 (1994) 752-756.

[14] Cone R., Proceedings, The frontiers of photospectral hole burning, Big Sky, Montana, mars 1997.

[15] Kohler B., Bernet S., Renn A. et Wild U., Opt. Lett. 18 (1993) 2144-46; Maniloff E.S., Altner S.B, Bernet S., Graf F.R., Renn A. et Wild U., Appl. Opt. 34 (1995) 4140-4148.

[16] Galaup J.-P., Grelet F., Le Gouët J.-L., Dolfi D. et Huignard, J.-P. demande de brevet FR 9802626.

[17] Lin H., Wang T. et Mossberg T. W., Opt. Lett. 20 (1995) 1658-1660.

[18] Babbitt W.R. et Mossberg T.W., J. Opt. Soc. Am. B 11 (1994) 1948-1953.

[19] Goodman J., Introduction à l'optique de Fourier et à l'holographie (Masson, Paris, 1972). 
[20] Rebane A., Opt. Commun. 67 (1988) 301-304.

[21] Anderson K. et Wagner K.H., postdeadline paper, Optics in Computing, Lake Tahoe, Nevada, mars 1997.

[22] Zhu M., Babbitt W.R. et Jefferson C.M., Opt. Lett. 20 (1995) 2514-2516.

[23] Lorgeré I., Rätsep M., Le Gouët J.-L., Grelet F., Tian M., Débarre A. et Tchénio P., J. Phys. B: At. Mol. Opt. Phys. 28 (1995) L565-L570; Rätsep M., Tian M., Lorgeré I., Grelet F. et Le Gouët J.-L., Opt. Lett. 21 (1996) 83-85; Le Gouët J.-L., Grelet F., Lorgeré I., Rätsep M., Tian M., Roblin M.-L. et Sigel C., Mol. Cryst. Liq. Crystal 291 (1996) 295-302. 